\title{
Inflammasomes induce sepsis following community breakdown
}

The intestinal microbiota has many beneficial roles but also contains potentially dangerous pathobionts. These pathobionts can cause severe disease when intestinal homeostasis is disturbed, but the mechanisms involved are not fully understood. Now, Ayres et al. have identified a pathobiont that is increased in number in the intestines of antibiotictreated mice and can trigger a fatal sepsis-like disease by activating the NAIP5 (neuronal apoptosis inhibitory protein 5)-NLRC4 (NOD-, LRR- and CARD-containing 4) inflammasome.

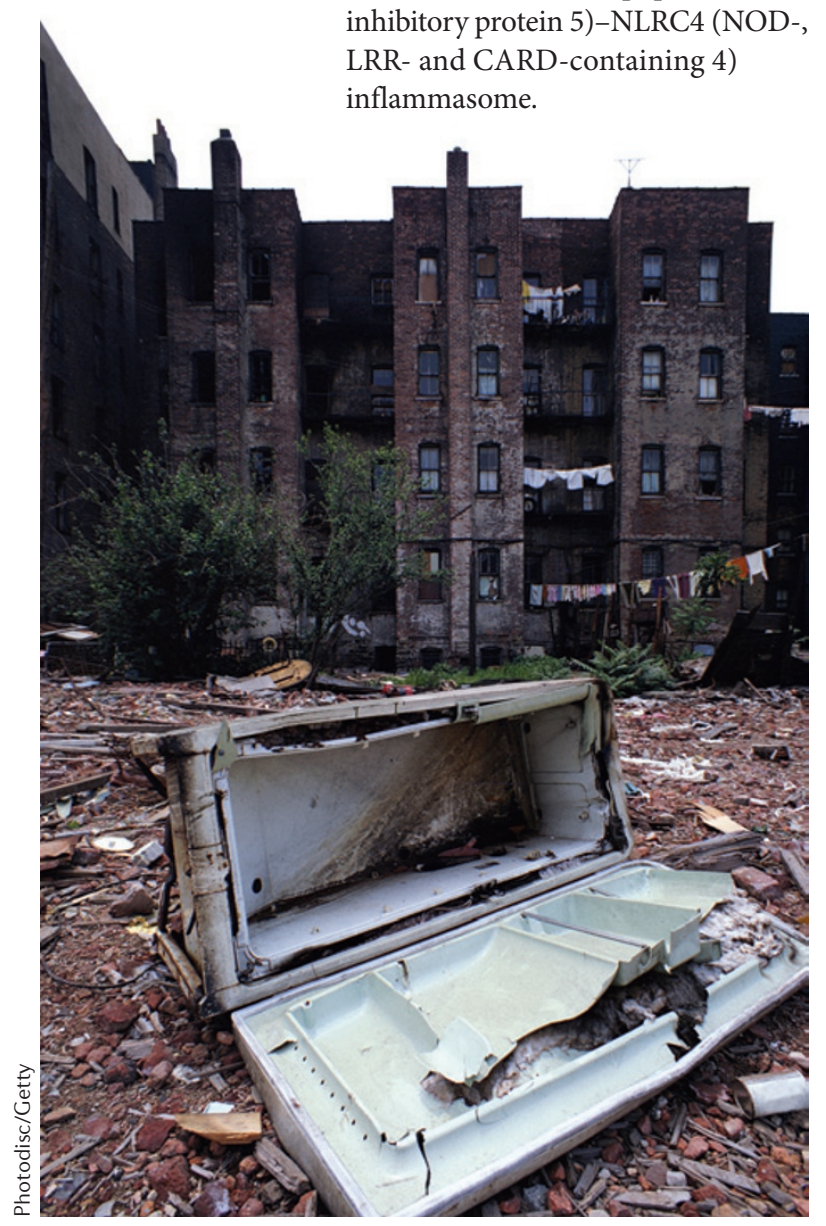

To disrupt the intestinal microbiota, the authors treated mice orally with the antibiotics ampicillin, vancomycin, neomycin and metronidazole (AVNM). Compared with untreated controls, AVNM-treated mice showed increased mortality in response to dextran sulphate sodium (DSS), a chemical that disrupts the colonic epithelium. Furthermore, whereas control mice developed colitis in response to DSS administration, AVNM-treated mice displayed a systemic sepsis-like disease, which was characterized by hypothermia and multiple organ failure.

Exploring the mechanisms behind the disease, the authors found that AVNM treatment resulted in overgrowth of AVNM-resistant bacteria in the intestinal tract. Following DSS administration, AVNM-resistant bacteria were also detected in the lungs and livers of AVNM-treated mice, but they were found at significantly lower levels in the lungs of the control DSS-only group and were not detected in the liver in these animals. The authors identified a specific Escherichia coli $\mathrm{O} 21: \mathrm{H}+$ isolate in the intestines of the mice in their colony that was expanded by AVNM treatment. Interestingly, this E. coli isolate could not be identified in mice that were obtained from the Jackson laboratory (JAX mice), and JAX mice did not show sepsis-like disease in response to AVNM and DSS treatment. However, following co-housing with the colony mice, JAX mice developed sepsis-like disease in response to AVNM and DSS treatment, suggesting that the E. coli $\mathrm{O} 21: \mathrm{H}+$ isolate and the disease it induces are transmissible.
Live systemic infection of mice with the E. coli $\mathrm{O} 21: \mathrm{H}+$ isolate induced a similar sepsis-like disease to that induced by AVNM and DSS treatment, providing further evidence that this isolate is a pathobiont. The authors sequenced the genome of the E. coli isolate and found that it encodes several potential virulence factors, including a functional flagellin and a type III secretion system. Such virulence factors have previously been shown to activate the NAIP5-NLRC4 inflammasome. Indeed, by using a retroviral lethality assay, the authors found that flagellin from the E. coli $\mathrm{O} 21: \mathrm{H}+$ isolate triggered macrophage death in an NAIP5- and NLRC4-dependent manner.

Finally, the authors showed that activation of the NAIP5-NLRC4 inflammasome promotes an interleukin-1 $\beta$-dependent sepsis-like pathology during systemic infection with the E. coli $\mathrm{O} 21: \mathrm{H}+$ pathobiont. Mice deficient for both NAIP5 and NLRC4 showed increased survival and attenuated disease during infection, despite having similar levels of extraintestinal bacterial colonization to wild-type controls. Taken together, these data describe a mechanism for how intestinal dysbiosis and epithelial disruption leads to sepsis. The authors propose that the inflammasome may be a useful therapeutic target in patients harbouring antibiotic-resistant pathobionts.

Yvonne Bordon

ORIGINAL RESEARCH PAPER Ayres, J. S.,

Trinidad, N. J. \& Vance, R. E. Lethal inflammasome activation by a multidrug-resistant pathobiont upon antibiotic disruption of the microbiota. Nature Med. 18, 799-806 (2012) 